\title{
A REMARK ON NILSSON TYPE INTEGRALS
}

\author{
NGUYEN SI MINH \\ Hanôi Institute of Mathematics \\ P.O. Box 631 Boho, Hanôi, Vietnam \\ BOGDAN ZIEMIAN \\ Institute of Mathematics, Polish Academy of Sciences \\ Śniadeckich 8, 00-950 Warszawa, Poland
}

\begin{abstract}
We investigate ramification properties with respect to parameters of integrals (distributions) of a class of singular functions over an unbounded cycle which may intersect the singularities of the integrand. We generalize the classical result of Nilsson dealing with the case where the cycle is bounded and contained in the set of holomorphy of the integrand. Such problems arise naturally in the study of exponential representation at infinity of solutions to certain PDE's (see [Z]).
\end{abstract}

1. Introduction. Nilsson type integrals were introduced by Nilsson $[\mathrm{N}]$ and their investigation was continued i.a. by Leray [L], Pham [P], Kobayashi [Ko], Andronikof [A] .

We are interested in analytic continuation of the function defined by integration of multivalued analytic functions depending on parameters. Let $f(z, \theta)$ be a given multivalued analytic function of variables $\theta \in \mathbb{C}^{n}$ with parameters $z \in \mathbb{C}^{m}$. We assume that there is a polynomial $P(z, \theta),(z, \theta) \in \mathbb{C}^{m} \times \mathbb{C}^{n}$, such that the singularities of $f(z, \theta)$ are defined by the equation $P(z, \theta)=0$ in $\mathbb{C}^{m} \times \mathbb{C}^{n}$. Let $\gamma(z)$ be a compact $n$-dimensional cycle in $\mathbb{C}^{n}$, depending continuously on the parameter $z \in \mathbb{C}^{m}$. Consider the Nilsson integral

$$
I(z)=\int_{\gamma(z)} f(z, \theta) d \theta .
$$

It is known $([\mathrm{N}],[\mathrm{L}],[\mathrm{A}])$ that, if $\gamma(z)$ does not intersect the set $V=\{P(z, \theta)=0\}$ for $z \in \mathbb{C}^{m}$ then $I(z)$ can be continued to $\mathbb{C}^{m}$ as a multivalued analytic function with singularities defined, in general, by the discriminants of the polynomial $P(z, \theta)$.

1991 Mathematics Subject Classification: Primary 32D; Secondary 32S.

This work was supported by the grant 210459101 from the Polish Scientific Research Committee.

The paper is in final form and no version of it will be published elsewhere. 
In this work, we study the same problem for the function $I(z)$ but in the case when $\gamma(z)$ may be unbounded and may intersect the singularities of the integrand $f(z, \theta)$. We consider the case where for $z \in \mathbb{C}, \gamma(z)=\stackrel{*}{a}+i \mathbb{R}^{n} \subset \mathbb{C}^{n}$ with $\stackrel{*}{a} \in \mathbb{R}^{n}$ fixed and $f(z, \theta)=$ $K(z, \theta) / P(z, \theta)$, where $P(z, \theta)$ is a polynomial of $(z, \theta)$ and $K(z, \theta)$ is a holomorphic function with some growth properties at infinity. We prove that $I(z)$ can be continued to $\mathbb{C}$ as a multivalued analytic function and its singularities can be described by "generalized discriminants" of the polynomial $P(z, \theta)$.

The first author wishes to express his thanks to the Institute of Mathematics of the Polish Academy of Sciences for its kind hospitality during the work on the problems considered in this paper.

2. Analytic continuation of $I(z)$. First let us recall some fact about Nilsson type integrals over intervals and unbounded cycle. Let $H(z, \theta)$ be a function of the complex variables $z=\left(z_{1}, \ldots, z_{k}\right) \in \mathbb{C}^{k}$ and $\theta \in \mathbb{C}$ such that there exists an algebraic variety $V_{H}$ in $\mathbb{C}^{k}$ of the form

$$
V_{H}=\left\{(z, \theta) \in \mathbb{C}^{k} \times \mathbb{C}: P(z, \theta)=0\right\},
$$

where $P$ is a nontrivial complex polynomial, such that $H(z, \theta)$ is holomorphic on the universal covering space $\mathbb{C}^{k+1} \backslash V_{H}$ of $\mathbb{C}^{k+1} \backslash V_{H}$. Recall (see [Z]) that if $P(z, \theta)$ is of the form

$$
P(z, \theta)=\sum_{j=0}^{m} a_{j}(z) \theta^{j}=a_{m}(z)\left(\theta-c_{1}(z)\right) \ldots\left(\theta-c_{m}(z)\right)
$$

then the polynomial

$$
\Delta_{\theta} P(z)=\left(a_{m}(z)\right)^{2 m-1} \prod_{j, l}\left(c_{j}(z)-c_{l}(z)\right)^{2}
$$

is called the complete discriminant of $P$ with respect to $\theta$. In the case where $P$ contains multiple factors the reduced discriminant of the polynomial $P, \widetilde{\Delta}_{\theta} P$, is obtained from $P$ by neglecting multiple factors.

We begin by stating the following reformulation of a lemma from $[\mathrm{N}]$ (Lemma 2, p. 466):

Lemma 1. Let $H(z, \theta)$ be as above and let $b^{1}(z)$ and $b^{2}(z)$ be algebraic functions on $\mathbb{C}^{k}$ such $\left(\stackrel{\circ}{z}, b^{1}(\dot{z})\right),\left(\stackrel{\circ}{z}, b^{2}(\dot{z})\right) \notin V_{H}$ for some $\stackrel{\circ}{z} \in \mathbb{C}^{k}$ and for all branches of $b^{1}$ and $b^{2}$. Then the function

$$
I(z)=\int_{b^{1}(z)}^{b^{2}(z)} H(z, \theta) d \theta
$$

defined in a neighbourhood of $z$, extends analytically to a multivalued holomorphic function on $\widetilde{\mathbb{C}^{k} \backslash V_{I}}$, where $V_{I}=\{z: W(z)=0\}$ with

$$
W(z)=\widetilde{\Delta}_{\theta} P(z) P_{1}(z) P_{2}(z) Q_{3}(z),
$$

where $\widetilde{\Delta}_{\theta} P(z)$ is the reduced discriminant of $P$ with respect to $\theta$, the $\operatorname{set}\left\{P_{j}(z)=0\right\}$ is 
the singular set of $b_{j}(j=1,2)$, and $Q_{3}$ is such that outside $\left\{Q_{3}(z)=0\right\}, P\left(z, b_{j}(z)\right) \neq 0$ for $j=1,2\left({ }^{1}\right)$.

We shall also consider the situation when either of the end points in (1) may be infinite (cf. Theorem 3.1 in $[\mathrm{Z}]$ ):

Lemma 2. Let $H$ be as above and let $\stackrel{*}{a} \in \mathbb{R}$. Fix $\stackrel{\circ}{z}=\stackrel{\circ}{\alpha}+i \stackrel{\circ}{\beta} \in \mathbb{C}^{k}$, and let $b(\beta)$ be a (real) algebraic function of $\beta \in \mathbb{R}^{k}$ such that

$$
P(\stackrel{\circ}{z}, \stackrel{*}{a}+i \gamma) \neq 0
$$

for $\gamma \leq b(\stackrel{\circ}{\beta})$. Define formally

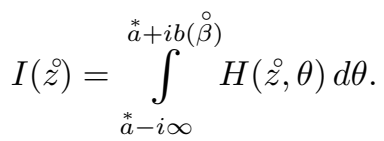

Suppose that

$$
|H(\stackrel{\circ}{z}, \theta)|=o(|\theta|) \quad \text { for } \operatorname{Re} \theta \geq \stackrel{*}{a} \text { with }|\theta| \text { large, }
$$

and locally uniformly in $z \in \mathbb{C}^{k}$,

$$
H(z, \theta) \leq C(z) \frac{1}{|\operatorname{Re} \theta|^{2}}
$$

for $\operatorname{Re} \theta$ positive and $\operatorname{Im} \theta$ bounded. Then the function I is well defined for $z=\stackrel{\circ}{\alpha}+i \beta$ with $\beta$ in some (real) open neighbourhood of $\beta$ in $\mathbb{R}^{k}$ and extends analytically to a multivalued function $I_{\alpha}(z)$ on $\mathbb{C}^{k} \backslash V_{I}$, where $V_{I}$ is defined by the polynomial $W$ as in Lemma 1 (with $\left.P_{1} \equiv 1\right)$. Explicitly, the analytic continuation of $I$ to the set

$$
\mathbb{C}^{k} \#\left(V_{I}+\overline{\mathbb{R}}_{+}^{k}\right):=\left(\mathbb{C} \backslash V_{I, 1}+\mathbb{R}_{+}\right) \times \ldots \times\left(\mathbb{C} \backslash V_{I, k}+\mathbb{R}_{+}\right)
$$

(the subscript standing for projection onto the respective $\mathbb{C}$ ) is given by the integrals

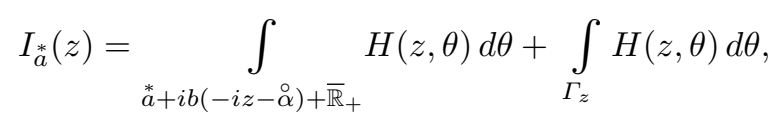

where for a fixed $z \in \mathbb{C}^{k} \#\left(V_{I}+\overline{\mathbb{R}}_{+}^{k}\right), \Gamma_{z}$ is a curve in $\mathbb{C}$ encircling the set $\{\theta: P(z, \theta)=$ $0\}+\overline{\mathbb{R}}_{+}$and contained in a small tubular neighbourhood of that set.

P r o of. It follows from Lemma 1 by deforming the contour of integration due to the estimates (2) and $\left(2^{\prime}\right)$.

Remark 1 . In the case where $b(\stackrel{\circ}{\beta}) \equiv+\infty$ and $H(z, \theta)=K(z, \theta) / P(z, \theta)$, where $P$ is a polynomial (without multiple factors) and $K$ is entire and such that the conditions (2) and $\left(2^{\prime}\right)$ are satisfied, we have

$$
I(z)=\sum_{j \in I^{+}(z)} \frac{K\left(z, c_{j}(z)\right)}{a_{m}(z) \prod_{q=1, q \neq j}^{m}\left(c_{j}(z)-c_{q}(z)\right)},
$$

$\left({ }^{1}\right)$ The existence of such $Q$ follows from the fact that the projection of an algebraic set is semialgebraic. 
where

$$
P(z, \theta)=a_{m}(z) \prod_{j=1}^{m}\left(\theta-c_{j}(z)\right)
$$

and

$$
I^{+}(z)=\left\{j: \operatorname{Re}, c_{j}(z)>\stackrel{*}{a}\right\} .
$$

For the proof of this proposition see [Z], Theorem 3.1.

We now consider the integral (to be understood in the sense of distributions if necessary)

$$
I(z)=\operatorname{reg} \int_{\substack{\prime * \\ a}+i \mathbb{R}^{n}} H(z, \theta) d \theta,
$$

where $\theta=\left(\theta_{1}, \ldots, \theta_{n}\right) \in \mathbb{C}^{n}, z \in \mathbb{C}, \stackrel{\prime *}{a}+i \mathbb{R}^{n} \subset \mathbb{C}^{n},{ }^{\prime} \stackrel{*}{a}=\left(\stackrel{*}{a}{ }_{1}, \ldots, \stackrel{*}{a}{ }_{n}\right) \in \mathbb{R}^{n}$.

Let $H(z, \theta)$ have the form

$$
H(z, \theta)=\frac{K(z, \theta)}{P(z, \theta)},
$$

where $P(z, \theta)$ is a polynomial of variables $(z, \theta) \in \mathbb{C} \times \mathbb{C}^{n}$, and $K(z, \theta)$ is an entire function. Set

$$
V_{H}=\{(z, \theta): P(z, \theta)=0\} .
$$

Fix $\stackrel{*}{a}_{0} \in \mathbb{R}$. This time, however, we admit that

$$
V_{H}\left(\stackrel{*}{a}_{0}, \stackrel{\prime *}{a}\right)=V_{H} \cap\left\{\left(\stackrel{*}{a}_{0}+i \mathbb{R}\right) \times\left(\stackrel{*}{a}^{\prime}+i \mathbb{R}^{n}\right)\right\} \neq \emptyset .
$$

For convenience we assume that $P$ has no multiple factors.

We start by considering the function

$$
H_{n-1}\left(z_{0}, \ldots, z_{n-1}\right)=\int_{a_{n}^{*}+i \mathbb{R}} H\left(z_{0}, \ldots, z_{n-1}, \theta\right) d \theta .
$$

The precise definition of $H_{n-1}$ requires however some caution:

For a fixed $\stackrel{*}{a}=\left(\stackrel{*}{a}_{0}, \ldots, \stackrel{*}{a}_{n}\right)$ we consider the system of two real algebraic equations in variables $\left(\beta_{0}, \ldots, \beta_{n}\right)$,

$$
\operatorname{Re} P(\stackrel{*}{a}+i \beta)=0, \quad \operatorname{Im} P(\stackrel{*}{a}+i \beta)=0 .
$$

Assuming that the system (7) is nondegenerate it describes an $(n-2)$-dimensional algebraic subset of $\mathbb{R}^{n}$. Then its projection onto the $n-1$ first variables is a semialgebraic set $A_{n-1}$ and we denote by $P_{n-1}\left(\beta_{0}, \ldots, \beta_{n-1}\right)$ a polynomial vanishing on that set. We solve the system (7) with respect to the variables $\left(\beta_{n-1}, \beta_{n}\right) \in \mathbb{R}^{2}$ with $\beta^{\prime \prime}=\left(\beta_{0}, \ldots, \beta_{n-2}\right)$ regarded as parameters. In particular, we obtain a multivalued algebraic function $\beta_{n-1}\left(\beta_{0}, \ldots, \beta_{n-2}\right)$ which clearly satisfies the equation

$$
P_{n-1}\left(\beta_{0}, \ldots, \beta_{n-1}\left(\beta_{0}, \ldots, \beta_{n-2}\right)\right)=0 .
$$

LEMma 3. For every point $\beta^{\prime}=\left(\beta_{0}, \ldots, \beta_{n-1}\right) \notin A_{n-1}$ the function $H_{n-1}\left(\ddot{a}+i \beta^{\prime}\right)$ defined by (6) in a neighbourhood of that point $\left(\right.$ in $\mathbb{R}^{n}$ ) extends to a multivalued function 
outside the set $\left\{\Delta_{n}=P\right\}$ given by the formula

$$
H_{n-1}\left(z^{\prime}\right)=\sum_{p \in I^{+}\left(z^{\prime}\right)} \frac{K\left(z^{\prime}, c_{p}\left(z^{\prime}\right)\right.}{a_{m}\left(z^{\prime}\right) \prod_{q=1, q \neq p}^{m}\left(c_{p}\left(z^{\prime}\right)-c_{q}\left(z^{\prime}\right)\right)},
$$

where $c_{j}$ and $I^{+}\left(z^{\prime}\right)$ are given by (3), (4) and $\Delta_{n} P$ is the discriminant of $P$ with respect to the variable $z_{n}$. Moreover, for any two points $\beta^{\prime}, \widetilde{\beta}^{\prime}$ belonging to the same open connected component of $\mathbb{R}^{n-1} \backslash A_{n-1}$ the corresponding extensions coincide.

Proof. The first part follows easily from Remark 1 since for $\beta^{\prime} \notin A_{n-1}, P\left(a^{*}+i \beta^{\prime}\right.$, $\left.\ddot{a}_{n}+i \gamma_{n}\right) \neq 0$ for $\gamma_{n} \in \mathbb{R}$. To see that the extensions coincide on the connected components of $\mathbb{R}^{n-1} \backslash A_{n-1}$ it is enough to check that the set $I^{+}$may change only for $\beta^{\prime}$ such that $\operatorname{Re} c_{j_{0}}\left(\ddot{a}^{\prime}+i \beta^{\prime}\right)=\stackrel{*}{a}_{n}$ for some $j_{0}$. But then we would have

$$
P\left(\stackrel{*}{a}^{\prime}+i \beta^{\prime}, \stackrel{*}{a}_{n}+i \operatorname{Im} c_{j_{0}}\right)=0,
$$

which means that $\beta^{\prime} \in A_{n-1}$ and proves the claim.

R e mark 2. The assertion of the lemma remains valid also when $P$ has multiple roots with the discriminant $\Delta_{n} P$ replaced by the reduced discriminant $\widetilde{\Delta}_{n} P$ (cf. [Z]) and with (7) replaced by a similar expression involving derivatives of $K$ with respect to $z_{n}$.

We next consider the function

$$
H_{n-2}\left(z_{0}, \ldots, z_{n-2}\right)=\int_{a_{n-1}+i \mathbb{R}} H_{n-1}\left(z_{0}, \ldots, z_{n-2}, \theta\right) d \theta .
$$

As before this integral requires explanation, especially that $H_{n-1}$ is not a single function but a family of multivalued functions. Thus $H_{n-2}$ is defined as follows:

Fix a point $\beta^{\prime \prime}=\left(\beta_{0}, \ldots, \beta_{n-2}\right) \in \mathbb{R}^{n-1}$ and let $\beta_{n-1}^{j}=\beta_{n-1}^{j}\left(\beta^{\prime \prime}\right), j=1, \ldots, m$, be the roots of $P_{n-1}\left(\beta^{\prime}\right)=0$ chosen so that

$$
-\infty=\beta_{n-1}^{0}<\beta_{n-1}^{1} \leq \ldots \leq \beta_{n-1}^{m}<\beta^{m+1}=\infty .
$$

For $\gamma_{n-1}, \beta_{n-1}^{j}<\gamma_{n-1}<\beta_{n-1}^{j+1}$ we are in one component of $\mathbb{R}^{n} \backslash A_{n-1}$ and we take the corresponding function $H_{n-1}$. We define

$$
H_{n-2}^{j}\left(\stackrel{*}{a}^{\prime \prime}+i \beta^{\prime \prime}\right)=\int_{\beta_{n-1}^{j}\left(\beta^{\prime \prime}\right)}^{\beta_{n-1}^{j+1}\left(\beta^{\prime \prime}\right)} H_{n-1}\left(\stackrel{* \prime \prime}{a}+i \beta^{\prime \prime}, \stackrel{*}{a}_{n-1}+i \gamma_{n}\right) d \gamma_{n} .
$$

In order that this be well-defined we must ensure that the singularities of $H_{n-1}$ do not lie on the interval of integration. The singularities of $H_{n-1}$ are described by the discriminant $\Delta_{n} P\left(\stackrel{*}{a}+i \beta^{\prime}\right)=0$, and we are thus led to the system of (real) equations

$$
\Delta_{n} P\left({ }^{* \prime}+i \beta^{\prime}\right)=0
$$

which replaces the system (7). We denote by $A_{n-2}$ the projection of $\left\{\Delta_{n} P=0\right\}$ onto the variables $\left(\beta_{0}, \ldots, \beta_{n-2}\right)$ and by $P_{n-2}\left(\beta_{0}, \ldots, \beta_{n-2}\right)$ the polynomial which describes $A_{n-2}$.

Lemma 4. For every fixed $\beta^{\prime \prime} \notin A_{n-2}$ and $j=0, \ldots, m+1$ the function $H_{n-2}^{j}\left(\stackrel{*}{a}^{\prime \prime}+i \beta^{\prime \prime}\right)$ defined initially in a neighbourhood of that $\beta^{\prime \prime}$, extends to a multivalued function outside 
the set $\left\{W_{n-2}\left(z^{\prime \prime}\right)=0\right\}$, where

$$
W_{n-2}=W_{n-1} \Delta_{n-1} \widetilde{P} Q_{n-2}, \quad W_{n-1}=\Delta_{n, n-1} P
$$

with

$$
\begin{aligned}
\widetilde{P}_{n-1}\left(z_{0}, \ldots, z_{n-2}, \beta_{n-2}\right) & =P_{n-1}\left(-i\left(z_{0}-\stackrel{*}{a}_{0}\right), \ldots,-i\left(z_{n-2}-\stackrel{*}{a}_{n-1}\right), \beta_{n-1}\right), \\
\Delta_{n, n-1} P & =\Delta_{n-1}\left(\Delta_{n} P\right),
\end{aligned}
$$

and $Q_{n-2}$ is the polynomial describing the set

$$
\Pi_{n-2}\left(\left\{\Delta_{n-1} P\left(z_{0}, \ldots, z_{n-2}, \stackrel{*}{a}_{n-1}+i \beta_{n-1}\right)=0\right\} \cap\left\{\widetilde{P}_{n-1}\left(z_{0}, \ldots, z_{n-2}, \beta_{n-1}\right)=0\right\}\right)
$$

whith $\Pi_{n-2}$ denoting the projection onto the variables $\left(z_{0}, \ldots, z_{n-2}\right)$. Moreover, the extension can be given by the (contour) integral

$$
H_{n-2}^{j}\left(z^{\prime \prime}\right)=\int_{\beta_{n-1}^{j}\left(-i\left(z^{\prime \prime}-\stackrel{*}{a}^{\prime \prime}\right),\right.}^{\beta_{n-1}^{j+1}\left(-i\left(z^{\prime \prime}-\stackrel{*}{a}^{\prime \prime}\right)\right)} H_{n-1}\left(z^{\prime \prime}, \stackrel{*}{a}_{n-1}+i \gamma_{n-1}\right) d \gamma_{n-1} .
$$

Pr o of. Clearly the proof consists in adapting Lemma 1 to our situation. We see that the function (10) extends to a holomorphic function in the complexifications $\zeta_{0}, \ldots, \zeta_{n-2}$ of the variables $\beta_{0}, \ldots, \beta_{n-2}$. Returning to the original variables $z$ by the formula $\zeta_{j}=$ $-i\left(z_{j}-\stackrel{*}{a}_{j}\right)$ we obtain (12). The ramification points of $H_{n-2}^{j}$ arise from the singular points of the integrand (described by $\Delta_{n, n-1} P=0$ ), the singular points of $\beta_{n-1}^{j}\left(-i\left(z^{\prime \prime}-\stackrel{*}{a}\right)\right.$ ) and of $\beta_{n-1}^{j+1}\left(-i\left(z^{\prime \prime}-\stackrel{*}{a}\right)\right)$ (described by $\left.\Delta_{n-1} \widetilde{P}_{n-1}=0\right)$ and the points $z^{\prime \prime}$ for which the integration limits are the singular points of the integrand (described by $Q_{n-2}=0$ ). This proves the lemma.

Remark 3. Clearly the function

$$
H_{n-2}=\sum_{j=0}^{m+1} H_{n-2}^{j}
$$

has the same regularity properties as $H_{n-2}^{j}$. Moreover, $H_{n-2}$ may be different on different components of the projection onto $\left(\beta_{0}, \ldots, \beta_{n-2}\right)$ of the sets $\mathbb{R}^{n} \backslash A_{n-1}$ minus the zero set $A_{n-2}$ of $P_{n-2}$.

By applying Lemma $4 n-1$ times we arrive at the following result for $I\left(z_{0}\right)=H_{0}\left(z_{0}\right)$ :

THEOREM 1. There exists a family of disjoint intervals $A_{0}^{1}, \ldots, A_{0}^{k}$ which fill the line $\stackrel{*}{a}_{0}+i \mathbb{R}$ up to a set of measure zero such that from each of these intervals the function $I\left(\stackrel{*}{a}_{2}+i \beta\right)$ extends to a ramified function on $\mathbb{C} \backslash\left\{W_{0}(z)=0\right\}$, where $W_{0}$ is defined by the recurrence $(11)$.

3. Example. We consider the polynomial

$$
P(z)=z_{1}^{2}+z_{2}^{2}+z_{3}^{2} \quad \text { in } \mathbb{C}^{3} .
$$

Let $\left.\stackrel{*}{a}=\left(\stackrel{*}{a}_{1}, \stackrel{*}{a}_{2}, \stackrel{*}{a}\right)_{3}\right) \in \mathbb{R}^{3}$ be fixed. For a fixed $b_{1} \in \mathbb{R}$ we shall solve the system of equations

$$
P(\stackrel{*}{a}+i b)=0
$$


with respect to the unknowns $b_{2}\left(\stackrel{*}{a}, b_{1}\right), b_{3}\left(\stackrel{*}{a}, b_{1}\right)$ with $\stackrel{*}{a}$ and $b_{1}$ regarded as fixed parameters. Explicitly, (13) is equivalent to the pair of equations

$$
\sum_{j=1}^{3}\left(\ddot{*}_{j}^{2}-b_{j}^{2}\right)=0, \quad \sum_{j=1}^{3} \stackrel{*}{a}_{j} b_{j}=0 .
$$

Suppose $a_{3} \neq 0$. Then $b_{3}=\left(1 / \stackrel{*}{a}_{3}\right) \sum_{j=1}^{2} \stackrel{*}{a}_{j} b_{j}$, and $\left(13^{\prime}\right)$ reduces to an equation in the unknown $b_{2}$ :

$$
A b_{2}^{2}+B b_{2}+C=0
$$

where

$$
\begin{aligned}
& A=\stackrel{*}{a}_{2}^{2}+\stackrel{*}{a}_{3}^{2}, \\
& B=2 \stackrel{*}{a}_{1} b_{1} \stackrel{*}{a}_{2}, \\
& C=\stackrel{*}{a}_{1}^{2} b_{1}^{2}-\stackrel{*}{a}_{3}^{2}\left(*_{1}^{2}+\stackrel{*}{a}_{2}^{2}+\stackrel{*}{a}_{3}^{2}-b_{1}^{2}\right) .
\end{aligned}
$$

The discriminant of (14) equals

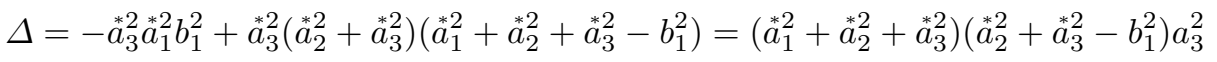

and is positive if and only if $\stackrel{*}{a}_{2}^{2}+\stackrel{*}{a}_{3}^{2}>b_{1}^{2}$. Hence if $\|*\|^{*} \|=\sqrt{*_{1}^{2}+\stackrel{*}{a}_{2}^{2}+\stackrel{*}{a}_{3}^{2}}$ and $\left\|^{\prime *}\right\|=$

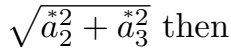

$$
\begin{aligned}
& b_{2}^{1}\left(\stackrel{*}{a}, b_{1}\right)=\frac{-2 \stackrel{*}{a}_{1} \stackrel{*}{a}_{2} b_{1}-\|* *\|^{*} \|^{\left\|^{\prime}\right\|^{*} \|^{2}-b_{1}^{2}}}{2\left\|\stackrel{a}{\prime}^{\prime}\right\|^{2}}, \\
& b_{2}^{2}\left({ }^{*}, b_{1}\right)=\frac{-2 \stackrel{*}{a}_{1} \stackrel{*}{a}_{2} b_{1}+\|\stackrel{*}{a}\| \sqrt{\left\|^{\prime *}\right\|^{2}-b_{1}^{2}}}{2\left\|^{\prime}\right\|^{*} \|^{2}} .
\end{aligned}
$$

So we fix $b_{1} \in \mathbb{R}$ such that $\left|b_{1}\right|<\left\|^{\prime *}\right\|$. We consider

$$
I\left(z_{1}\right)=\int_{a^{\prime}+i \mathbb{R}^{2}} \frac{K\left(z_{1}, \theta_{2}, \theta_{3}\right)}{P\left(z_{1}, \theta_{2}, \theta_{3}\right)} d \theta_{2} d \theta_{3} .
$$

Let $c\left(z_{1}, \theta_{2}\right)=\sqrt{-z_{1}-\theta_{2}^{2}}$ so that

$$
P\left(z_{1}, \theta_{2}, \theta_{3}\right)=\left(\theta_{3}-c\left(z_{1}, \theta_{2}\right)\right)\left(\theta_{3}+c\left(z_{1}, \theta_{2}\right)\right) .
$$

Since for $z_{1}=\stackrel{*}{a}_{1}+i b_{1}$ and $\theta_{2}=\stackrel{*}{a}_{2}+i b_{2}$ with $b_{2} \neq b_{2}^{1}\left(\stackrel{*}{a}, b_{1}\right), b_{2}^{2}\left(\stackrel{*}{a}, b_{1}\right)$,

$$
P\left(z_{1}, \theta_{2}, \stackrel{*}{a}+i b_{3}\right) \neq 0 \quad \text { for } b_{3} \in \mathbb{R}
$$

we may compute the integral

$$
H\left(z_{1}, \theta_{2}\right)=\int_{a_{3}^{*}+i \mathbb{R}} \frac{K\left(z_{1}, \theta_{2}, \theta_{3}\right)}{P\left(z_{1}, \theta_{2}, \theta_{3}\right)} d \theta_{3} .
$$

by the residue theorem and obtain, as in $\S 8$ of $[\mathrm{Z}]$,

$$
H\left(z_{1}, \theta_{2}\right)= \begin{cases}\frac{K\left(z_{1}, \theta_{2}, c\left(z_{1}, \theta_{2}\right)\right)}{2 c\left(z_{1}, \theta_{2}\right)} & \text { for } b_{2}>b_{2}^{2}\left({ }^{*}, b_{1}\right), \\ 0 & \text { for } b_{2}^{1}\left(\stackrel{*}{a}, b_{1}\right)<b_{2}<b_{2}^{2}\left(\stackrel{*}{a}, b_{1}\right), \\ -\frac{K\left(z_{1}, \theta_{2},-c\left(z_{1}, \theta_{2}\right)\right)}{2 c\left(z_{1}, \theta_{2}\right)} & \text { for } b_{2}<b_{2}^{1}\left(\stackrel{*}{a}, b_{1}\right) .\end{cases}
$$


Hence

$$
\begin{aligned}
& I\left(z_{1}\right)=-\int_{a_{2}^{*}-i \infty}^{\stackrel{*}{a}_{2}+i b_{2}^{1}\left(\stackrel{*}{a}, b_{1}\right)} \frac{K\left(z_{1}, \theta_{2},-c\left(z_{1}, \theta_{2}\right)\right)}{2 c\left(z_{1}, \theta_{2}\right)} d \theta_{2} \\
& +\int_{a_{a}^{*}+i b_{2}^{2}\left(\stackrel{*}{a}, b_{1}\right)}^{{\stackrel{*}{a_{2}}+i \infty}^{*}} \frac{K\left(z_{1}, \theta_{2},-c\left(z_{1}, \theta_{2}\right)\right)}{2 c\left(z_{1}, \theta_{2}\right)} d \theta_{2}
\end{aligned}
$$

Now in order to investigate the regularity of the function $I\left(\stackrel{*}{a}_{1}+i b_{1}\right)$ with respect to $b_{1}$ we must see when the singular points of the integrand lie on the integration half-lines. The singular points are given by the equation $z_{1}^{2}+\theta_{2}^{2}=0$, and by setting $z_{1}=\stackrel{*}{a}{ }_{1}+i b_{1}, \theta_{2}=$ $\stackrel{*}{a}_{2}+i \gamma_{2}$ we find the solutions

$$
b_{1}=-\stackrel{*}{a}, \gamma_{2}=\stackrel{*}{a} 1 \text { and } b_{1}=\stackrel{*}{a}_{2}, \gamma_{2}=-\stackrel{*}{a}{ }_{1} .
$$

Denote by $A_{1}, A_{2}, A_{3}$ the intervals $\left(-\left\|^{\prime} \stackrel{*}{a}\right\|,-\stackrel{*}{a}_{2}\right),\left(-\stackrel{*}{a}_{2}, \stackrel{*}{a}_{2}\right),\left(\stackrel{*}{a},\left\|^{\prime} \stackrel{*}{a}\right\|\right)$, respectively. Then it follows from Lemma 2 that for each $A_{j}$ there exist $\varepsilon_{j}, \widetilde{\varepsilon}_{j} \in\{+,-\}$ such that for $\beta_{1} \in A_{j}, I$ can be written as (cf. Example in $\S 8$ of $[\mathrm{Z}]$ )

$$
\begin{aligned}
I\left(\stackrel{*}{a}_{1}+i b_{1}\right)= & \int_{\mathbb{R}_{+}} \frac{K\left(z_{1}, \varepsilon_{j} i z_{1},-\widetilde{\varepsilon}_{j} c\left(z_{1}, \varepsilon_{j} i z_{1}+\gamma\right)\right)}{2 \widetilde{\varepsilon}_{j} c\left(z_{1}, \varepsilon_{j} i z_{1}+\gamma\right)} d \gamma \\
& -\int_{\mathbb{R}_{+}} \frac{K\left(z_{1}, \varepsilon_{j} i z_{1},-\widetilde{\varepsilon}_{j} c\left(z_{1}, \varepsilon_{j} i z_{1}+\gamma\right)\right)}{2 \widetilde{\varepsilon}_{j} c\left(z_{1}, \varepsilon_{j} i z_{1}+\gamma\right)} d \gamma \\
& -\int_{a_{2}+i b_{2}^{1}+\mathbb{R}_{+}} \frac{K\left(z_{1}, \varepsilon_{j} i z_{1},-\widetilde{\varepsilon}_{j} c\left(z_{1}, \varepsilon_{j} i z_{1}+\gamma\right)\right)}{2 \widetilde{\varepsilon}_{j} c\left(z_{1}, \varepsilon_{j} i z_{1}+\gamma\right)} d \gamma \\
& +\int_{a_{2}^{*}+i b_{2}^{2}+\mathbb{R}_{+}} \frac{K\left(z_{1},-\varepsilon_{j} i z_{1}, \widetilde{\varepsilon}_{j} c\left(z_{1},-\varepsilon_{j} i \zeta_{1}+\gamma\right)\right)}{2 \widetilde{\varepsilon}_{j} c\left(z_{1},-\varepsilon_{j} i z_{1}+\gamma\right)} d \gamma \\
& +\int_{\mathbb{R}_{+}} \frac{K\left(z_{1},-\varepsilon_{j} i z_{1}, \widetilde{\varepsilon}_{j} c\left(z_{1},-\varepsilon_{j} i z_{1}+\gamma\right)\right)}{2 \widetilde{\varepsilon}_{j} c\left(z_{1},-\varepsilon_{j} i z_{1}+\gamma\right)} d \gamma \\
& -\int_{\mathbb{R}_{+}} \frac{K\left(z_{1},-\varepsilon_{j} i z_{1},-\widetilde{\varepsilon}_{j} c\left(z_{1},-\varepsilon_{j} i z_{1}+\gamma\right)\right)}{2 \widetilde{\varepsilon}_{j} c\left(z_{1},-\varepsilon_{j} i z_{1}+\gamma\right)} d \gamma .
\end{aligned}
$$

Now by Lemma 2 the first, second, fifth and sixth integrals extend to functions in $z_{1}$ with a ramification point at $z_{1}=0$. Hence in particular if $\stackrel{*}{a}_{1}>0$ they are holomorphic if $\operatorname{Re} z_{1} \geq$ $\stackrel{*}{a}_{1}$. The case of the third and fourth integrals is slightly more delicate. Considering each of them as a function in the variable $b_{1} \in A_{j}$ (and not in $z_{1}=\stackrel{*}{a}+i b_{1}$ ) we derive again from Lemma 1 that they extend to ramified functions in the variable $\zeta_{1}$ (= complexification of $\left.b_{1}\right)$ with ramification points at $\zeta_{1}=i a_{1}^{*}$ and at $\zeta_{1}= \pm \zeta_{1}= \pm\left\|^{\prime} a\right\|^{*} \|$. Now introducing back the variable $z_{1}$ in these extensions by the formula $\zeta_{1}=-i\left(z_{1}-\stackrel{*}{a}{ }_{1}\right)$, we obtain ramified functions in $z_{1}$ with ramification points at $z_{1}=0$ and $z_{1}=\stackrel{*}{a}_{1} \pm i\left\|^{\prime} \stackrel{*}{a}\right\|$. Thus the desired extensions assume the form 


$$
\begin{aligned}
& -\int_{a_{a}^{*}+i b_{2}^{1}\left(a_{1}^{*},-i\left(z_{1}-\stackrel{*}{a}_{1}\right)\right)+\mathbb{R}_{+}} \frac{K\left(z_{1}, \varepsilon_{j} i z_{1},-\widetilde{\varepsilon}_{j} c\left(z_{1}, \varepsilon_{j} i z_{1}+\gamma\right)\right)}{2 \widetilde{\varepsilon}_{j} c\left(z_{1}, \varepsilon_{j} i z_{1}+\gamma\right)} d \gamma \\
& +\int_{a_{2}^{*}+i b_{2}^{2}\left(\stackrel{*}{a}_{1},-i\left(z_{1}-a_{1}^{*}\right)\right)+\mathbb{R}_{+}} \frac{K\left(z_{1},-\varepsilon_{j} i z_{1}, \widetilde{\varepsilon}_{j} c\left(z_{1},-\varepsilon_{j} i z_{1}+\gamma\right)\right)}{2 \widetilde{\varepsilon}_{j} c\left(z_{1}-\varepsilon_{j} i z_{1}+\gamma\right)} d \gamma
\end{aligned}
$$

\section{References}

[A] E. Andronikof, Intégrales de Nilsson at faisceaux constructibles, Bull. Soc. Math. France 120 (1992), 51-85.

[Ko] T. Kobayashi, On the singularities of solutions to the Cauchy problem with singular data in the complex domain, Math. Ann. 269 (1984), 217-234.

[L] J. Leray, Le calcul différentiel et intégral sur une variété analytique complexe, ibid. 87 (1959), 81-180.

[N] N. Nilsson, Some growth and ramification properties of certain multiple integrals, Ark. Mat. 5 (1965), 463-476.

[P] F. Pham, Singularités des systèmes différentiels de Gauss-Manin, Birkhäuser, 1981.

[Z] B. Ziemian, Leray residue formula and asymptotics of solutions to constant coefficient PDEs, Topol. Methods Nonlinear Anal. 3 (1994), 257-293. 